\title{
MEJORAMIENTO DEL HÁBITAT DESDE LA EDUCACIÓN POPULAR
}

\section{Guillermo Javier Marzioni}

Arquitecto. Facultad de Arquitectura, Diseño y Urbanismo. Universidad Nacional del Litoral. Magíster en Hábitat y Vivienda. Universidad Nacional de Mar del Plata. Se ha desempeñado en Asociación Civil Canoa, Fundación Vivienda y Comunidad, Subsecretaría de Desarrollo Urbano y Vivienda de la Nación y actualmente en Comisión Nacional de Tierras para el Hábitat Social. guimarzio@hotmail.com; marzionig@jgm.gov.ar

ISNN 1666-6I86. Volumen I3 N I3 (Octubre 20I2) pp. 09I-III - Recibido: I6-03-II.Aprobado: I8-08-I2. 


\section{Resumen}

Este texto está basado en algunos fragmentos de la tesis "Mejoramiento del Hábitat desde la perspectiva de la Educación Popular de Paulo Freire", orientada por Víctor Saúl Pelli, que se publicará con el nombre Hábitat popular, encuentro de saberes. El tema investigado es la coincidencia de métodos y propósitos entre las acciones de asistencia al mejoramiento del hábitat y el enfoque de la Educación Popular, en la particular concepción del educador Paulo Freire. Se describe a la Educación Popular en un recorte vinculado con el interés de este estudio. Luego, se da cuenta de definiciones y experiencias de hábitat popular profundizando especialmente en el aspecto habitacional. Posteriormente, con el objetivo de elaborar el cruce temático, se indaga en materiales teóricos precedentes entre los escritos de referentes empiristas y académicos acerca de cómo el mejoramiento del hábitat popular en asentamientos espontáneos se vincula con los procesos educativos propuestos por el pedagogo brasileño Paulo Freire.

\section{Palabras clave}

Educación, hábitat, vivienda,_opresión, participación (Tesauros de UNESCO).

\section{Abstract}

This text is based on excerpts from the thesis "Housing Improvement from the perspective of Popular Education of Paulo Freire", edited by Victor SAUL PELLI to be published under the title "Popular Habitat, a Melding of Different Sources of Knowledge".. The research topic is the matching of methods and purposes between relief actions to improve habitat and a popular education approach, in the specific conception of Paulo Freire, the educator. Popular Education is described selectively so as to coincide with the interest of this study. The study then proposes definitions of habitat and experiences of deepening popular education especially in the housing dimension. Later, with the goal of developing a cross thematic approach, previous theoretical writings are investigated, as expressed by empiricists and academics, in order to understand how the improvement of popular habitat in spontaneous settlements can be linked to the educational processes proposed by Paulo Freire.

\section{$\underline{\text { Keywords }}$}

Education, habitat, housing, oppression, participation (UNESCO Thesaurus). 


\section{REVISIÓN DE LAS TEORÍAS SOBRE EL MEJORAMIENTO DEL HÁBITAT DES- DE LA EDUCACIÓN POPULAR}

$\mathrm{Al}$ analizar algunos procesos de mejoramiento del hábitat popular se observa que estos parten del respeto por los saberes de los habitantes, sus acumulaciones y sus riquezas. De este modo, es posible afirmar que incorporan, explícita o implícitamente, conceptos y metodologías concordantes con los de la Educación Popular de PAUlo Freire.

Los investigadores en el hábitat abarcan en sus estudios y experiencias cuestiones como metodologías de abordaje participativas, búsqueda de tecnologías adecuadas y apropiadas, respeto por los saberes y modos culturales de los habitantes y la intención —en el momento de iniciar las acciones sobre el hábitat - de contemplar las inspiraciones, aspiraciones, intereses y deseos de los que habitan.

Estas dimensiones fueron trabajadas por FreIRE y "los equipos de los círculos de cultural" para posibilitar la intervención en la educación de adultos. La mirada compleja de la Educación Popular que se centra en el sujeto opresor-oprimido con el que se establece un diálogo transformador plantea una integralidad que puede ayudar a intervenir en el hábitat.

Entonces, en este texto, se intentará la identificación de espacios, modos de coincidencia y aporte recíproco entre los procedimientos de intervención en el hábitat popular - particularmente en el mejoramiento habitacional de los asentamientos espontáneos - y los postulados y métodos de la Educación Popular.

\section{LA EDUCACIÓN POPULAR EN FREIRE}

En un momento internacional de ebullición y cambios populares, se construye en América Latina un movimiento social e intelectual que puede sintetizarse en la idea del "hombre nuevo" y que incide en FrEIRE y sus contemporáneos. Esta idea tiene su correlato en distintas disciplinas, tales como la política, la sociología, el arte, la economía, la teología, la arquitectura y el urbanismo, en el que se encuentran los teóricos y hacedores del hábitat popular en los que puede observarse una sincronicidad con las primeras ideas sobre Educación Popular. Vale señalar que la obra de FrEIRE se desarrolla entre 1965 y 1995.

En este contexto surgen los conceptos de Educación Popular. Luego, a partir de reconocer la realidad de las barriadas pobres de las grandes ciudades, comienzan a expresarse y difundirse conceptos — que venían acuñándose desde décadas anteriores— sobre el trabajo integral en el mejoramiento del hábitat. $\overline{1-\text { Los círculos de cultura son }}$ grupos de alfabetización de adultos, iniciados en Brasil por FREIRE en la década del 60. Se utiliza la expresión Educación Popular en letras mayúsculas al referirse a la "escuela" de PAULO FREIRE y para distinguirla de otras ideas y miradas de pedagogos que también enmarcan su acción y teoría desde la educación popular. 
La relación de dominantes y dominados que instalaron ENGELS y MARX es retomada por Freire (Freire, 1985 (2): 231), quien complejiza el concepto poniendo en evidencia la parte de dominante que todo dominado tiene dentro de sí.

Solo cuando los oprimidos descubren nítidamente al opresor y se comprometen en la lucha organizada por su liberación, empiezan a creer en sí mismos, superando así su complicidad con el régimen opresor.

En el método aplicado por FreIRE en los años 60 se trabaja con la población adulta con "Situaciones" (FREIRE, 1985 (3): 34), cartillas dibujadas con preguntas disparadoras para comprender la relación del hombre con el mundo, la naturaleza y la cultura.

El hombre hace el pozo porque tiene necesidad de agua. Y lo hace en la medida en que, relacionándose con el mundo, hace de él objeto de su conocimiento y lo somete, por el trabajo, a un proceso de transformación. Así hace la casa, su ropa, sus herramientas.

La comprensión de FREIRE sobre "El sujeto en la Realidad" provee materiales para entender el involucramiento del hombre con el ambiente construido, con su contexto. En los sectores populares el habitante es autoconstructor de su vivienda, de su barrio, de su hábitat, y es artífice de la construcción de la realidad.

El hacer para resolver lo inmediato y urgente es un ejercicio permanente en el campo popular. Podría decirse que luego del tránsito por alguna de las experiencias de su vida cotidiana, el habitante (o la comunidad) comienza a plantearse profundamente ciertas cuestiones y complejiza su mirada sobre la realidad. Este es el momento en que se produce el salto cualitativo del que habla Freire: "Sé ahora que soy culto', afirmó enfáticamente un viejo campesino. Y al preguntarle cómo lo sabía, respondió con el mismo énfasis: 'Porque trabajo y trabajando transformo al mundo'." (FreIRE, 1985: 107).

Esta conciencia crítica es una experiencia que también atraviesan los educadores en el ejercicio de esta nueva mirada sobre la realidad del campo popular; así lo expresa el pedagogo brasileño: "Lo que ocurre en el medio popular, en las periferias de las ciudades, en el campo - en los trabajadores urbanos y rurales que se reúnen para demandar o para discutir sus derechos - nada de esto puede escapar de la aguda curiosidad de los educadores dedicados a la práctica de la educación popular”. (FreIRE, 1985: 31). 
Mejoramiento del hábitat desde la educación popular

La dimensión política de las prácticas es un aporte que trae la Educación Popular a la tarea cotidiana de la educación de adultos e influye, como pensamiento, en otras áreas.

Esta búsqueda de la verdad en el diálogo de los saberes aparece en FreIRE como camino hacia la liberación. En el trabajo grupal se integra la relación con el mundo y se propone la problematización para despertar de la opresión. "De este modo se quiebran los argumentos de 'autoridad': ya no hay alguien que eduque a otro" (FrEIRE, 1985 (2): 104).

Como bien lo señala en Pedagogía del Oprimido, es necesario atravesar las asimetrías en las relaciones para entablar un encuentro de saberes; romper con la autodesvalorización de los sujetos a quienes se orienta un programa o una acción; desactivar la idea del profesional como quien sabe - y a quien se debe escuchar - y repensar los criterios del saber impuestos por los convencionales academicismos. “(...) cierta vez, escuchamos decir a un líder campesino, en una reunión de una de las unidades de producción —un asentamiento de la experiencia chilena de reforma agraria-: 'Nos decían que no producíamos porque éramos borrachos, perezosos. Toda mentira. Ahora, que somos respetados como hombres vamos a demostrar a todos que nunca fuimos borrachos ni perezosos. Éramos explotados, eso sí', concluyo enfáticamente." (FrEIRE, 1985 (2): 105).

Para que el encuentro de saberes pueda construirse, una herramienta indispensable es la participación. Es por esta razón que se la estimula en los programas o proyectos que intervienen en el mejoramiento del hábitat. Para FrEIRE, la participación expresa el crecimiento que los sujetos van teniendo tanto en el plano individual como en el de la transformación de la realidad.

\section{REPASO HISTÓRICO SOBRE EL HÁBITAT}

\subsection{La cuestión del hábitat}

En la Primera Cumbre del Hábitat, de 1976, se sintetizaron las redefiniciones del problema y las búsquedas de soluciones que habían emprendido distintos investigadores y quienes realizaron experiencias populares en las barriadas de las ciudades del mundo, particularmente en las latinoamericanas que habían crecido de modo vertiginoso en esa última década (HARDOY, 1987: 13). La Declaración sobre los Asentamientos Humanos (UN Hábitat, 1976) fue tomada por la Comisión de Hábitat de las Naciones Unidas, creada poco antes, como uno de los principales documentos que traza el mandato del organismo y que dejó establecido el Derecho a una Vivienda Adecuada en el marco del problema del hábitat. 
Desde ese momento, nuevas metodologías de abordaje se intentan y replican en el ámbito internacional. Incorporan una concepción más compleja y de muy distinto signo que las propuestas urbanísticas de los Congresos Internacionales de la Arquitectura Moderna, basadas en la tabula rasa y en el supuesto de una sociedad cultural y económicamente homogénea. De esta manera, se intenta dar cabida a los crecientes problemas de la pobreza, influyendo en las acciones de los gobiernos locales y multiplicándose en numerosas organizaciones sociales. La Segunda Cumbre del Hábitat realizada en Estambul, en 1996, fue la oportunidad de observar las mejores prácticas efectuadas en el sentido conceptual y metodológico planteado en Hábitat I. Un hito importante, en el planteo de estas políticas, es el Foro Urbano Mundial (Nairobi, 2002), que abre la participación de las organizaciones populares a través de una instancia orgánica permanente bianual de carácter consultivo en la Comisión de las Naciones Unidas para el Hábitat, reconociéndose el rol protagónico de los habitantes.

En referencia a estos planteos internacionales se han establecido derechos internacionales dedicados al acceso de la población a la Tierra, la Vivienda y el Hábitat, planteados en acuerdos y pactos, luego refrendados en Argentina y la mayoría de los países, así como los derechos constitucionales y la legislación específica que enmarca el accionar en relación con los asentamientos espontáneos. Estos derechos otorgan legalidad a la legitimidad que tiene la resolución popular del problema habitacional, que muchas veces no coincide con las formas convencionales de la sociedad y del mercado. Estos marcos jurídicos constitucionales desactivan la idea de La Ciudad Legal y la Ciudad Ilegal sobre la que avanzó el Instituto de Investigación y Estudios para Desarrollo (HARDOY, 1987: 26).

\subsection{Debates sobre el problema habitacional}

A partir de la idea de reconocer la existencia y el valor social e inmobiliario de los asentamientos humanos espontáneos (o no planificados), comienza a teorizarse sobre ellos. Se ponen en evidencia, en algunos autores, los pilares teóricos de un debate que aporta a la discusión sobre el valor de lo existente basado en la ponderación de aquellas construcciones que, con grandes esfuerzos, los habitantes del lugar fueron realizando en procesos de larga acumulación.

En los fundamentos del presente trabajo, algunos de los textos de referencia están vinculados con la corriente marxista en la que puede encuadrarse la obra de FrEIRE - que a su vez incorpora otras variables - y con otras concepciones de una sociedad más justa. Entre ellas se encuentra la vertiente propositiva del anarquismo, de la que abreva JoHN 
Mejoramiento del hábitat desde la educación popular

TURNER, quien dio impulso a la idea de valorizar y recuperar lo producido aun en condiciones poco favorables. En este sentido, se entiende la vivienda como un proceso social. Este camino conduce hacia las ideas de Emilio Pradilla referidas a la vivienda popular, en un encuentro - que refleja polémicas estructurales de mayor amplitud- entre la teoría marxista y otras versiones del ideario socialista, como la concepción anarquista. En este contexto resultan imprescindibles las contribuciones y complejidades de la mirada de la Educación Popular para detenerse en los planteos que parten de recuperar los aportes que realizan los sujetos sociales, superando los obstáculos de una sociedad con desigualdades. Se presenta una definición construida por el director de la Maestría de Hábitat y Vivienda (FERNÁNDEZ WAGNER, 1998: 35): "La palabra vivienda, por tanto, debería emplearse más en su acepción de 'proceso' que como unidad de habitación. Los verdaderos valores son aquellos basados en las relaciones entre los elementos que intervienen en la vivienda: los actores, sus actividades y sus logros.”

Esta idea de proceso proviene de la tradición instalada en el campo de lo teórico sobre la cuestión habitacional por el arquitecto inglés JOHN TURNER. Proveniente de un país con una tradición estatal en políticas de construcción de viviendas para los trabajadores y de regulación del mercado inmobiliario, TURNER, al observar las barriadas de las ciudades latinoamericanas, valora la acción de los habitantes frente a la desprotección del Estado. La reflexión que realiza desde su experiencia sobre autoconstrucción en Arequipa y cuenta en el artículo "Vivienda en Tres Dimensiones" permite explicar el término (TURNER, 1978). "El proceso de vivienda -incluyendo a ahorrar para ello, ocupando tierra para ello, construyendo y reconstruyendo o mejorándolo, gerenciándolo y manteniéndolo, amoblándolo - tiene en cuenta una proporción substancial de trabajo y capital".

TURNER introduce también el análisis de la vivienda desde el punto de vista del usuarioproductor y no de un consumidor final de un producto. El usuario aparece como gestor o constructor, tal como se produce la vivienda en el proceso de urbanización latinoamericano (FERNÁNDEZ WAGNER, 1998: 61).

La crítica al trabajo de TURNER en el campo teórico es de Emilio Pradilla, quien se apoya en los planteos de ENGELS sobre la necesidad de resolver los problemas estructurales de los ingresos de los "obreros pauperizados y los componentes del ejército de reserva" (Pradilla, 1974: 293), para luego resolver la cuestión habitacional. La cuestión de la desigualdad es abordada por TURNER desde el análisis de lo prioritario y lo posible en la perspectiva del lapso de vida del poblador. 
Las reflexiones de ENGELS sobre la cuestión de la vivienda — surgidas del pensamiento de la Europa Central en el período de la Revolución Industrial — aportan fundamento ideológico para la profundización y desarrollo de la problemática habitacional. Dice en Contribución al problema de la Vivienda, cuya primera edición es de 1872 (ENGELs, 1980: 18): "Para acabar con esta penuria de vivienda no hay más que un medio: abolir la explotación y la opresión de las clases trabajadoras por las clases dominantes".

El planteo de ENGELS va a la profundidad de las causas generadoras del problema de la vivienda, anclado en la desigualdad social y en las condiciones económicas de mercado que determinan su rentabilidad y que están permitidas por ciertas formas jurídicas reconocidas socialmente.

Este pensamiento crítico y el de MARX fueron las bases de los planteos que el pueblo, a través del movimiento social (en su forma gremial, por ejemplo), esgrimió durante todo el siglo XX, estadio de verdadero crecimiento del poder popular en el que los ciudadanos organizados pudieron adquirir derechos avalados por pronunciamientos internacionales. Estos logros alcanzados por el pueblo protegieron a las clases trabajadoras, en el marco del Derecho, frente al devenir histórico de los acontecimientos que provocaron el crecimiento económico y la acumulación del capital en pocas manos, particularmente en las ciudades. La penuria de vivienda en América Latina, en África o en Asia muestra que no fue suficiente este avance y que queda mucha deuda social por saldar.

Estos sectores obreros, en las realidades de desocupación y exclusión masiva y permanente del tercer mundo actual, se pueden incluir en la denominación de sectores populares o simplemente trabajadores.

La posición de Emilio Pradilla es que los trabajadores, que también son los autoconstructores de su vivienda, reproducen su condición de oprimidos. Asimismo sostiene que el valor de una vivienda por autoconstrucción es muy alto si se considera el aporte de trabajo que efectúa el usuario. "(...) autoconstruirán su vivienda, también antihigiénica, insalubre, insegura y hacinada, que será a la vez símbolo de sus condiciones de explotación por el capital". (PRADILlA, 1974: 293).

Es muy difícil traducir esta imagen a la realidad latinoamericana de la segunda mitad del siglo XX, en la que los trabajadores no son obreros sino desocupados, socialmente excluidos o "nunca ocupados", y en la que el autoconstructor no cae en manos de un explotador 
visible, sino que no encuentra sustento digno en un sistema que lo excluye e ignora. Sin embargo, es un aporte muy valioso para reflexionar sobre los mecanismos de denuncia que se tienen que poner en funcionamiento para la liberación de los trabajadores frente a la opresión del mercado y las clases dominantes vigentes.

Se toma entonces del planteo de Pradilla su encuadre ideológico, ya que este permite expresar la contradicción de opresores y oprimidos, dada la base marxista de sus teorías. Opresores y Oprimidos es uno de los ejes que se toma también en este trabajo para comprender la Educación Popular.

Se revisan, asimismo, los conceptos de PRADILLA sobre la autoconstrucción porque no puede desconocerse la realidad de todo el ambiente construido o autoconstruido en las ciudades. Esta cuestión se podrá poner en valor en el momento de implementar políticas de intervención, no para idealizar los asentamientos espontáneos, sino para reconocerlos y trabajar en su transformación hacia el mejoramiento, salvando la brecha de las desigualdades. El encuadre elegido en este trabajo convoca a la transformación social para realizar un movimiento que permita el acceso a la tierra y a la vivienda higiénica, salubre, segura y espaciosa, en el cual se considera a la autoconstrucción como una opción elegida y no obligada por la circunstancia económica.

En este sentido, se vuelve sobre los textos de PRADILla porque son necesarios para comprender que si se elige la autoconstrucción y el mejoramiento de los asentamientos espontáneos como respuesta posible en el contexto latinoamericano, es a sabiendas de la desigualdad social imperante. Y este es un dato de la realidad presente en los países empobrecidos de contextos económicos neoliberales, donde los sectores dominantes manejan plenamente el juego de las relaciones del mercado y, en consecuencia, de la sociedad. Pero no se abandona la idea de que estas relaciones, sin embargo, pueden transformarse.

Se destaca la visión de Pradilla como continuador del pensamiento marxista que deja establecida con claridad la existencia de una diferenciación social entre opresores y oprimidos, a diferencia de otros autores que entienden el problema de la pobreza como un dato de la realidad que se acepta sin cuestionar sus causas de injusticia en la distribución de las riquezas. El otro aspecto por el que se recupera a PRADILLA es su calidad de conocedor del hábitat de los trabajadores, posición que lo alinea a ENGELS y a su histórico Informe sobre la situación de la clase obrera en Inglaterra (ENGELS, 1843), y a sus propuestas de generación de política habitacional desde la responsabilidad que corresponde al Estado 
en Contribución al problema de la vivienda (ENGELS, 1872). Es importante aclarar que la referencia a PRADILLA en este libro se basa en sus fundamentos y no en el detalle de forma de la solución habitacional o el tipo de propuesta. Es decir, interesa su concepción del rol indelegable del Estado en la distribución de la riqueza a través de las políticas públicas que inciden directa e indirectamente en el mejoramiento del hábitat.

En tanto Víctor Pelli, con particular preocupación y desempeño sobre el mejoramiento del hábitat en los asentamientos espontáneos, encuentra en el tema de la autoconstrucción la clave para el abordaje de la realidad presente de Latinoamérica.

En las diversas versiones de autoconstrucción, “(...) en todas ellas se plantea la inclusión de los habitantes en el proceso de producción de su propia solución habitacional. Este núcleo es sólido y marca una profunda diferencia con todas las propuestas de gestión habitacional que excluyen al habitante del proceso de resolución (y que continúan gozando de la predilección de los sectores dominantes)". (PeLli, 2007: 93).

La intervención del "Estado como redistribuidor del poder con criterios de equidad" (PELLI, 2007: 149) será necesaria para el acompañamiento del mejoramiento del hábitat, contemplando procesos de autoconstrucción con la aplicación de "soluciones alternativas" a través de políticas públicas que provoquen la distribución de la riqueza y del poder cívico en el país y transformen la opresión en liberación de los sectores populares.

\subsection{El concepto de hábitat popular}

En los años 80, se comenzó a hablar de hábitat popular, fundamentalmente en América Latina. Aquí se transcribe el concepto de elaboración colectiva que surge de un taller en Colombia: "El hábitat popular es un conjunto de aspectos necesarios para el adecuado desarrollo de la vida familiar y social de la población, haciendo hincapié en las capas de menores recursos, que son las que tienen mayores dificultades para cubrir esas necesidades. Esto significa incluir en el concepto no solo el usufructo de una vivienda adecuada, sino también el de los servicios físicos y sociales imprescindibles, así como la cobertura de las necesidades de trabajo, alimentación salud, educación y esparcimiento, que también son básicas" (BORUCHALSKI, 1986: 2).

Este concepto que circuló en América Latina fue apropiado en la Argentina por diversas organizaciones, grupos universitarios y movimientos que desarrollaban trabajos en esa línea. 


\subsection{La idea de producción social del hábitat}

A mediados de los años 90, desde las organizaciones sociales, se ha revisado el concepto de hábitat popular atravesándolo con otras variables. Se plantea tomar dimensión de las actividades que se desarrollan en ese hábitat popular para su concreción. Esto es: la producción de materiales, los modos de comercialización y financiación, las complejidades de gestión y las acumulaciones que el pueblo tiene en sus estrategias cotidianas y que permiten la consolidación habitacional en los asentamientos. "Por Producción Social del Hábitat se entiende '... todos aquellos procesos generadores de espacios habitables, componentes urbanos y viviendas, que se realizan bajo el control de auto productores y otros agentes sociales que operan sin fines lucrativos. Pueden tener su origen en las propias familias actuando individualmente, en grupos organizados informales, en empresas sociales como las cooperativas y asociaciones de vivienda, o en las Organizaciones No Gubernamentales, los gremios profesionales e incluso las instituciones de beneficencia que atienden emergencias y grupos vulnerables." (ORTIZ FlORES, 2006: 4).

\subsection{El mejoramiento habitacional en asentamientos espontáneos}

El artículo de Víctor Pelli sobre el mejoramiento habitacional en asentamientos espontáneos (PelLi, 1998: 45) detalla con precisión estos términos, que abarcan básicamente dos cuestiones: la vivienda y el barrio en un contexto de espontaneidad que implica no planificación y autoconstrucción.

El reconocimiento global de las situaciones habitacionales espontáneas es una "forma germinal de solución" (Pelli, 1998: 45). El autor indica aspectos positivos de las soluciones habitacionales espontáneas: su naturaleza autogestionaria; el manejo preciso y realista de las prioridades; la flexibilidad normativa y la agilidad para las transacciones en el mercado formal e informal.

Para el momento de elegir la radicación de un asentamiento espontáneo es quizás el término consolidación el que deja más clara la acción que está expresando: dejar mejor plantado, más firme o soldado lo que se realizó previamente. En tanto la expresión mejoramiento es la que más se va instalando en la comunidad y en las políticas públicas, razón por la cual se deja planteada aquí como la palabra para seguir utilizando y promoviendo.

Como en este trabajo se intenta abordar el problema del mejoramiento en los asentamientos espontáneos en todo el país, y en el contexto latinoamericano, se utiliza el término 
asentamiento propuesto por el arquitecto Víctor Pelli (Pelli, 1995: 57), considerando incluidas las villas.

El nivel de precariedad de las viviendas en los asentamientos espontáneos urbanos no está dado solo por la precariedad de los materiales, sino por la falta de servicios e infraestructura y por el problema de la "Seguridad de la Tenencia de la Tierra" (NACIONES UnIDAS HÁBITAT, 2006). En este sentido, el mejoramiento habitacional en estos barrios necesita incluir la dimensión jurídico-legal del problema de la tierra, así como también el ordenamiento urbano dominial.

La meta que se persigue en el objetivo de mejorar la vivienda existente es lograr una "vivienda digna" o, mientras este concepto no se clarifique, la "vivienda socialmente aceptable". Cuando una comunidad o un país acuerden sobre cuál es el estándar socialmente aceptable, se dejará el camino allanado para la definición de políticas de resolución del problema habitacional.

En la definición de Diseño Participativo se comprende a las metodologías de abordaje en procesos de mejoramiento del Hábitat como instancias interdisciplinarias, multiactorales, concertativas e intersectoriales. El momento de diseño no es solo la acción de los profesionales o técnicos con la participación de los habitantes, sino una comunión de ideas y propuestas que se crean conjuntamente; esta es la clave del abordaje desde la Educación Popular.

La Planificación Participativa a escala de ciudad tiene una dimensión muy diferente respecto de la del mejoramiento participativo de asentamientos espontáneos. Esto atañe a las estrategias, los métodos, los recursos y los actores. Sin embargo, se considera valioso traer este aporte porque cuando el abordaje del mejoramiento del hábitat toma dimensiones más allá de la vivienda individual se está irrumpiendo en la ciudad. Entonces, entran en el juego del mejoramiento habitacional o del barrio actores que responden a otros intereses ajenos a los de los habitantes. Los asentamientos espontáneos surgen y se consolidan en áreas urbanas más amplias, y la intervención sobre ellos requiere interconexiones con el resto de la ciudad. También es aplicable la idea de planificación participativa en el interior de asentamientos que por su escala superan el tamaño de algunas ciudades.

La participación de la población en los procesos de diseño de la ciudad responde a postulados ciertamente nuevos del urbanismo pensado desde las instituciones estatales. Los 
Mejoramiento del hábitat desde la educación popular

procesos populares cada vez están más presentes en las toma de partido sobre nuevas obras y, en particular, cuando los habitantes están involucrados directamente.

3.6. La decisión del mejoramiento habitacional y del hábitat

Víctor Pelli, en el análisis de los modelos de vivienda llave en mano y mejoramiento habitacional, afirma que es más amplio el alcance de las soluciones centradas en las necesidades básicas. Es decir: más habitantes mejoran su situación habitacional si se parte de las soluciones parciales y se postergan las soluciones de vivienda completa. Propone, en función de esto, trabajar prioritariamente sobre las acciones de construcción gradual de satisfactores básicos, entre las cuales se encuentran las de mejoramiento habitacional. “... trabajar con niveles parciales de satisfacción de necesidades habitacionales básicas, como escalones en un proceso de evolución gradual, para una mayor cantidad de personas o para la totalidad de la población en situación crítica dentro de una jurisdicción y de un plazo razonable en relación con la duración de la vida” (Pelli, 2007: 201).

Esta conceptualización puede dejar sentada la ideología de una política habitacional y ser un insumo para el diseño e intervención de programas habitacionales. "Estas acciones de satisfacción parcial pueden materializarse, en correspondencia con el señalamiento de posibles necesidades básicas (críticas), en elementos habitacionales (situaciones, condiciones, bienes o servicios) de fuerte impacto en la situación de habitabilidad, tales como: provisión de acceso a red de agua potable, provisión de terrenos en mejores condiciones de ubicación urbana o territorial, regularización dominial en terrenos ocupados, provisión de núcleos sanitarios o 'pies de casa' (construcciones parciales con característica que facilitan la evolución posterior), ampliaciones o mejoramientos parcial de la vivienda existente, o mejoramiento de vías publicas de acceso" (PELLI, 2007: 201).

Sobre los programas que comprenden en sus objetivos niveles parciales de satisfacción $\mathrm{y}$, fundamentalmente, aquellos que parten de mejorar lo construido existente, se va incorporando con mayor presencia el rol del Estado. "Como dice Kliksberg: 'Empieza a reconocerse que los Estados son los únicos que pueden proveer un conjunto de condiciones esenciales para el desarrollo económico: ley, orden, políticas macroeconómicas efectivas, desarrollo de la infraestructura, inversión en capital humano y mejoramiento de la equidad" (PÉrez Coscio, 1999: 45).

En lo que respecta a la política habitacional del país, se ha construido una tradición sobre el mejoramiento del hábitat en estas tres últimas décadas, desde las primeras experiencias 
piloto realizadas puntualmente hasta la instrumentación de programas estatales difundidos en algunas ciudades o en todo el país. Algunos de estos programas lograron continuidad en el tiempo atravesando varias etapas gubernamentales, han tenido muy buena aceptación en la población y han sido reconocidos internacionalmente. Sin embargo, en lo que respecta al presupuesto representan un porcentaje bajo respecto del total de fondos destinados al problema habitacional. Y las obras, en su mayoría, son ejecutadas a través de empresas aun en tiempos en que el gobierno ha puesto especial énfasis, y valores récord de inversión, en la política de vivienda con destino a los sectores populares.

\section{CRITERIOS DE LA EDUCACIÓN POPULAR DETECTABLES EN LA TEORÍA Y LA PRÁCTICA DE LA INTERVENCIÓN EN EL HÁBITAT}

En diversas cuestiones planteadas por la comunidad de investigadores y organizaciones vinculadas con el hábitat popular reaparecen conceptos trabajados por FrEIRE, o de similar encuadre teórico e ideológico. Están en juego en la búsqueda de soluciones para la satisfacción de las necesidades de los sectores populares y también en el fortalecimiento grupal para obtener mayor capacidad autogestionaria. Estas acciones estimulan el crecimiento del sujeto y lo impulsan a ser protagonista indispensable del propio cambio, adquiriendo conciencia de derechos y capacidad para la resolución de los problemas.

En los asentamientos espontáneos existe la necesidad de resolver lo inmediato y cotidiano. Pero mientras se van cubriendo algunas necesidades básicas del habitar, se crece en organización y en conciencia de que los planteos - no solo como sujetos individuales, sino como sujetos colectivos - permiten ir elevando la voz. En algunas oportunidades, los habitantes expresan sus demandas comunitarias en la forma de un "proyecto", exigiendo, como parte de la sociedad y del Estado, una respuesta. En general se identifican áreas o programas sociales orientados a la resolución de necesidades del sector y se establecen mecanismos de gestión colectiva. Estas acciones de las organizaciones sociales requieren la incorporación de nuevos aprendizajes para el desempeño en el mejoramiento del hábitat.

Para que las instancias de participación tengan los tópicos de la Educación Popular, la capacitación, la formación política y el acompañamiento a las organizaciones participantes son necesarios, para que eleven el nivel de conciencia crítica y puedan plantear sus necesidades no solo como reclamo, sino como reivindicación, y construyan un nuevo conocimiento transformador. 
4.1. La mirada integradora de FREIRE en el encuentro de saberes

Se toman algunas frases de FREIRE en la acción de la educación que ayudan a comprender su mirada integradora sobre la relación entre el que trae un conocimiento y el que tiene un conocimiento previo. Luego se observa cómo esta relación se da en la acción del hábitat al sondear en la producción teórica de los autores que escriben sobre este. "Basta ser hombre para ser capaz de captar los datos de la realidad. Basta ser capaz de saber, aun cuando sea un saber meramente vulgar. De ahi que no haya ignorancia absoluta ni sabiduría absoluta. Nadie ignora todo, nadie sabe todo. La absolutización de la ignorancia, además de ser la manifestación de una conciencia ingenua de la ignorancia y del saber, es un instrumento del que se sirve la conciencia dominadora para arrastrar a los llamados 'incultos', los 'absolutamente ignorantes' que 'incapaces de dirigirse', necesitan de la 'orientación', de la 'dirección', de la 'conducción' de los que se consideran a sí mismos 'cultos y superiores'.' (FreIRE, 1985: 101).

En esta perspectiva, se quiebran los argumentos de "autoridad", ya que no hay alguien que eduque a otro, sino que ambos, a través del diálogo, lo hacen en comunión. La idea de comunión se asemeja a la de horizontalidad, expresada a menudo en la comunidad de los que trabajan en hábitat.

"El educador no podrá entonces 'apropiarse' del conocimiento, sino que este será solo aquello sobre lo cual educador y educando reflexionen” (FREIRE, 1985: 106).

Aquí la apelación más cercana a los temas de hábitat es la del diseño participativo, que más adelante se amplía.

4.2. El diálogo como construcción de conocimiento en el mejoramiento del hábitat El abordaje al problema del mejoramiento del hábitat requiere la articulación permanente de los actores y la predisposición para comprender los diversos planteos, las pujas de intereses sectoriales y políticos entre los que se encuentran los intereses de los habitantes. El debate se establece acerca de quién tiene la verdad sobre la acción que se pretende realizar. Pero para lograr encarar estas acciones se necesita llegar a acuerdos, que solo podrán surgir a partir del establecimiento de un diálogo.

La disponibilidad para lo que FreIRE llama "dialoguicidad", la predisposición del uno para relacionarse con el otro y el sentido de humildad que significa rescindir mi cuestión para dar lugar a la cuestión planteada por el otro forman parte de un ejercicio que permite construir conjuntamente. 
Para llevar adelante un proyecto en el que se plantea la participación real de todas las partes, se hace necesario contar con una actitud que permita despojarse de los conocimientos previos para aprender más. Dar cabida a la palabra del otro es la condición básica para su conocimiento. En los proyectos de hábitat, se ha trabajado sobre esta idea y particularmente sobre la posibilidad de que los habitantes pueden hablar para que se conozcan sus intereses. Por citar un ejemplo, en Los Que Habitan Tienen La Palabra (BerretA, 1989: 24) se deja planteada esta cuestión.

Será necesario instrumentar técnicas de participación para que la palabra de los distintos pueda expresarse; es decir, para que surja la palabra de quienes se encuentran con mayores dificultades para lograr sus planteos debido a una historia de opresión que instaló una asimetría que deberá salvarse si se quiere lograr un verdadero intercambio de ideas.

4.3. La participación como metodología de abordaje al problema del hábitat

Se indagan aquí los elementos coincidentes con la Educación Popular que expresan los autores dedicados al tema del hábitat. Se toman particularmente los textos de Víctor Pelli, quien destina gran parte de su producción teórica a comprender el vínculo entre los que habitan y los que intervienen sobre el hábitat popular. Este autor resalta la idea de que el protagonismo en la gestión del hábitat incluye a quienes habitan, así como FreIRE dedica su preocupación a la relación educador-educando y a las complejidades de esa interrelación. "El centro y la motivación genuina de la acción social de la vivienda se encuentran en las necesidades de la gente." (PELLI, 2007: 15).

En estos protagonistas, que FreIRE llama sujetos sociales, se centra el objeto de la acción del hábitat que se quiera emprender. "Toda elaboración, de acción, o de decisión politica, sobre la solución habitacional, debe tener su basamento en el conocimiento profundo y constantemente actualizado de las necesidades de las personas, entendido como conocimiento construido junto con la misma gente que las padece, no solo la de las estadísticas y de las encuestas, sino las personas concretas, con nombres, rostros y diálogos, una por una, uno por uno." (PeLLI, 2007: 16).

La noción de sujeto de FreIRE aparece en palabras como "rostros, nombres, una, uno", actualizando PELLI en la expresión "una por una" la mirada de género que FREIRE incorporó a su lenguaje en los últimos años.

Luego, se refiere a las condiciones de reaseguro que necesitan darse para que la participación colabore en romper las asimetrías de poder en las que los sectores sociales oprimidos 
Mejoramiento del hábitat desde la educación popular

se encuentran respecto de los otros, y lograr, de esa manera, que se lleve adelante un proceso de concertación o (dicho en términos de FrEIRE) de dialoguicidad.

4.4. El diseño participativo, metodología de Educación Popular

Al diseñar, construir, planificar o gestionar participativamente viviendas, un proyecto o una obra (infraestructura, un grupo de viviendas o mejoramientos habitacionales o el salón comunitario), técnicos y habitantes parten del conocimiento de su realidad en busca de la resolución de objetivos comunes.

Para que puedan provocarse instancias de integración se necesita crear momentos de trabajo adecuados y preparados para ello (BORUCHALSKI, 1986), en los que el equipo no solo proponga una técnica, sino que también se involucre en ese encuentro para que de allí surja la idea. Estas tareas, en tanto realizadas conjuntamente entre técnicos y usuarios, se pueden entender como equivalentes al acto de educar, porque aparece la necesaria transferencia de conocimientos de las especificidades de los diversos saberes técnicos, pero también de los saberes que los habitantes traen de experiencias anteriores o actuales y la información directa que incorporan a partir de estar más involucrados en los problemas que acontecen en su organización, barrio, ciudad o región donde se esté realizando el proyecto.

En la Asociación Civil Canoa se define (GALlo, 1989: 2) que el encuentro de saberes se compone de la interrelación de diferentes capacidades técnicas, científicas y empíricas. Esto constituye interdisciplina en tanto se reconoce la especificidad de cada saber disciplinar para luego relacionarse con el otro. Entre estos distintos saberes se incorpora el saber popular.

Se presenta a continuación una definición de diseño participativo elaborada por la arquitecta María Bernabella Pelli, en la que se plantea la idea de proceso de acción participativa. "El diseño participativo, de acuerdo a como lo entendemos, no es un método de 'manual', pre elaborado, que se aplica como receta en proyectos (aunque podría existir una guía orientadora de la actividad), es una herramienta que se construye y se desarrolla dentro de espacios de acción participativa, a lo largo de un proceso social que está fuertemente orientado y pautado por un marco teórico determinado.” (Pelli, B.; 2004).

4.5. Los habitantes de asentamientos actuando en el mejoramiento del hábitat

Los habitantes de los asentamientos se constituyen en nuevos sujetos sociales territoriales actuando en el hábitat como líderes barriales o como habitantes que definen sus derechos en su epopeya cotidiana tras el planteo de sus necesidades básicas no resueltas. A partir 
de lo expuesto, se señalan algunos interrogantes: ¿para quién queda el espacio de representación que se estructura en la democracia representativa? ¿Es este un lugar posible de acceso para los sectores populares? Cuando estos sujetos sociales - construidos desde el territorio- se plantean la irrupción en lo político, surge un vector de análisis entre las prácticas populares y las políticas estatales. Es para destacar que en este ejercicio de gestión colectiva los compañeros de los barrios se transforman en sujetos politizados y se proponen trascender su accionar más allá del espacio de lo barrial, ampliando su territorialidad y siendo, en ocasiones, partícipes en estructuras del Estado.

\section{CONCLUSIÓN}

En referencia a la comparación teórica se deja constancia de que en los escritos sobre hábitat se encuentran coincidencias con los textos de la Educación Popular.

La Educación Popular tiene un desarrollo de ideas que aporta elementos teóricos para comprender las prácticas en el hábitat. Esta mirada puede sumarse a la de quienes realizan el esfuerzo de observar y escribir sobre el problema habitacional, tradición que se trae desde ENGELS.

La actuación en el mejoramiento del hábitat conlleva aprendizajes colectivos basados en la experiencia de la vida cotidiana y en las tecnicidades propias de disciplinas urbanísticas que provocan una integración o encuentro de saberes.

Los compañeros y compañeras habitantes de los asentamientos protagonizan su accionar como sujetos sociales territoriales y se constituyen en sujetos políticos como individuo y como colectivo a partir de la experiencia que les otorga la participación en el mejoramiento del hábitat. Esta acción funda ciudadanía porque los sujetos se transforman en ciudadanos a partir de una práctica de gestión, en pos de la mejora del barrio, a través de la cual desarrollan una mirada más compleja del mundo.

La asociatividad en el ámbito de lo local, regional e internacional de los grupos que bregan por el Derecho a la tierra, la vivienda y el hábitat abre espacios para la construcción de una mirada crítica de la realidad y, a través de ella, para una visión del mundo desde el pueblo y hacia su liberación. 
La mirada de la Educación Popular en la historia de los pueblos aporta herramientas para comprender el trabajo en el hábitat popular. La Educación Popular es una perspectiva política para el abordaje al mejoramiento de los asentamientos espontáneos y no un cruce de temas.

La Educación Popular parte de entender que el saber popular es valioso, y a partir de esto recupera y construye, buscando nuevos elementos en un proceso dialéctico. Como se plantea en el inicio de la investigación, se puede comprobar en los textos consultados y en las experiencias analizadas que el mejoramiento de lo existente en el hábitat popular - en los casos en que se encara con determinados criterios - se vincula con el encuadre conceptual de los procesos de Educación Popular promovidos por PAULO FreIRE.

Observando desde la teoría de FREIRE, se puede señalar que en algunas acciones de hábitat popular el técnico asume el rol del educador y el habitante asume el de sujeto de la acción de educación, y ambos confluyen en un plano de igualdad en la construcción del saber, en la construcción del hábitat.

Los asentamientos espontáneos en la mayoría de las ciudades de los países latinoamericanos (como en otros territorios donde el pueblo soporta la dominación de países dominantes o de su propia oligarquía) necesitan del apoyo del Estado para que sus habitantes puedan obtener el acceso definitivo al Derecho a la Tierra, la Vivienda, el Hábitat, la Ciudad.

Existen buenos ejemplos, para replicar, de programas y acciones gubernamentales —con apoyo de organizaciones sociales - que apuntan a mejorar las barriadas. Si la intervención en los asentamientos espontáneos recupera las construcciones existentes, esto conlleva un reconocimiento de los saberes de los autoconstructores, lo cual constituye un encuentro de saberes, como lo plantea FrEIRE, que implica también incorporar la participación plena de los habitantes en todas las fases del proceso de mejoramiento de su hábitat. 


\section{BIBLIOGRAFÍA}

BERRETA, Horacio (1989) Los que habitan tienen la palabra. CEVE (Ed.), Córdoba. BORUCHALSKI, Mario; BOTTERO, José (1986). “Taller Latinoamericano sobre Hábitat Popular". Centro de Estudios del Hábitat Popular - Programa Estudios de Vivienda para América Latina. Mimeo. Medellín.

ENGELS, Federico (1980) Contribución al problema de la Vivienda. (1. a Ed. 1872).

FERNÁNDEZ WAGNER, Raúl (1998). "El debate académico de los 1970 a 1980". En: Teorías en Hábitat y Vivienda (Pág. 35). (Ed.) General Pueyrredón, Universidad de Mar del Plata.

FREIRE, Paulo (1985) Pedagogía del Oprimido. (1. ${ }^{a}$ Ed. 1970) SIGLO XXI, Buenos Aires. FREIRE, Paulo (1992) La educación como práctica de la libertad (1. ${ }^{\text {a }}$ Ed. 1969). Siglo XXI, Buenos Aires. El libro integra un texto de 1965. Aclaración: escrito en Santiago, Chile. GALLO, Sandra y otros (1989). Cartilla Institucional de Canoa. Santa Fe.

HARDOY, Jorge y SATTERTHWAITE, David (1987) La Ciudad Legal y La Ciudad Ilegal. Latinoamericano (Ed.), Buenos Aires.

MARZIONI, Guillermo (2010). "Mejoramiento del Hábitat desde la perspectiva de la Educación Popular de Paulo Freire”. Tesis de Maestría en Hábitat y Vivienda. FAUD UNMP (Ed.), Mar del Plata.

MARZIONI, Guillermo (2012) Hábitat popular encuentro de saberes. Nobuko (Ed.), Buenos Aires.

ORTIZ FLORES, Enrique (2006). Producción Social del Hábitat. Componente estratégico de las políticas de Estado. XV Asamblea de Ministros de Urbanismo y Vivienda, Montevideo.

PRADILLA, Emilio (1974). Pradilla. E. (1996) Autoconstrucción, explotación de la fuerza de trabajo y políticas de Estado en América Latina. UNMP (Ed.). En: Teorías en Hábitat y Vivienda. Universidad de Mar del Plata. (Ed.) General Pueyrredón.

PELLI, María Bernabella (2008) Construcción y Desarrollo de Espacios Participativos de Diseño. Máster en Gestión Social del Hábitat. Escuela Técnica de Arquitectura. Mimeo. Sevilla.

PELLI, Víctor (1995) “El mejoramiento habitacional de los asentamientos espontáneos”. En: Cuaderno 2 de la Cátedra Sociología Urbana. FADU UNNE. Resistencia.

PELLI, Víctor (1998). “El mejoramiento habitacional de los asentamientos espontáneos”. En: Gestión Habitacional. Maestría de Hábitat y Vivienda. FAUD UNMP (Ed.), Mar del Plata. 
Mejoramiento del hábitat desde la educación popular

PELLI, Víctor (2007). Habitar, Participar, Pertenecer. Nobuko. (Ed.), Buenos Aires. PÉREZ COSCIO, Luis (1999) (compilador) Calidad, fortalecimiento y sostenibilidad en los programas de créditos y asistencia para el mejoramiento habitacional. IIED AL, Subsecretaría de Vivienda de Nación (Ed.), Buenos Aires.

TURNER, John (1998) "Vivienda en tres dimensiones: términos de referencia para una redefinición de la cuestión de la vivienda" (1. a publicación de 1978). En: Teorías en Hábitat y Vivienda. Universidad de Mar del Plata (Ed.), General Pueyrredón.

NACIONES UNIDAS HÁBITAT (1976) Declaración de los Asentamientos Humanos. Vancouver. www.unhabitat.org:80/declarations/vancouver.asp.

NACIONES UNIDAS HÁBITAT (2006) Seguridad en la Tenencia de la Tierra. www. unhabitat.org. 